Bull. Austral. Math. Soc.

06A12, 90A07

VOL. 37 (1988) [379-382]

\title{
ON SAFETY AND RIVALS IN POWER-STRUCTURES
}

\author{
JOHN BORIS MILlER
}

\begin{abstract}
It is shown that every minimal power-structure other than the singleton power-structure has at least two contenders; that is, any contender has at least one rival. The safe core of a power-structure is empty if and only if the power-structure is minimal.
\end{abstract}

'Nessun dorma'-Turandot.

The theory of coups d'état in power-structures, introduced by the author in $[1]$, represents an organisation as a finite lattice $(X, \leqslant)$, and a power-structure within the organisation as a convex $\vee$-subsenulattice $B$ of $X$. The boss of the power-structure is $\vee B$, the lattice supremum of the subset $B$. A coup occurs in $B$ when a subset $T$ of $B$ containing $\vee B$ is removed from $B$ so as to leave a subset $B \backslash T$ which again is a power-structure (the surviving power-structure of the coup), the cardinality of $T$ being minimal for all possible choices of such subsets. (The removal of $T$ from $B$ is metaphorical only; the sets $X, B$ and the lattice structure are not to be thought of as changing at some point of time.) The set $T$ is called the topple set of the coup, and completely characterises the coup; its cardinality $|T|$ is called the stability of the power-structure $B$, written $t(B)$. The member $\vee(B \backslash T)$ is the boss of the surviving power-structure. A given power-structure may admit more than one coup. Any boss $c$ of a surviving power-structure is called a contender of $B$; the coup is said to promote c. Any member $d$ of $B$ who is covered by the boss of $B$ (that is, $d<\vee B$, and $d<x<\vee B$ for no $x \in B$, hence for no $x \in X)$ is called a deputy of $B$.

Introduce the notation $(\leqslant a)$ for the set $\{x \in X: x \leqslant a\}$. It was shown in [1] that every contender $c$ is necessarily a deputy; and that the topple set of the coup promoting $c$ is unique, $=B \backslash(\leqslant c)$. Thus the stability of $B$ is

$$
t(B)=|B \backslash(\leqslant c)|=\min \{|B \backslash(\leqslant d)|: d \text { is a deputy of } B\} .
$$

A minimal power-structure is a power-structure $B$ which is minimal among all power-structures having the same boss and stability as $B$. That is, if $\mathcal{P}$ denotes the set of all power-structures of the organisation $X$, then $B$ in $\mathcal{P}$ is called minimal if for all $C \in \mathcal{P}, C \subset B$ and $\vee C=\vee B$ imply $t(C)<t(B) .(\subset$ denotes strict inclusion.) In this note we prove the

Received 24 July 1987

Copyright Clearance Centre, Inc. Serial-fee code: 0004-9729/88 \$A2.00+0.00. 
THEOREM OF THE RIVAL. Every minimal power-structure other than a singleton power-structure has at least two contenders.

This theorem was stated without proof in [1]. To quote the interpretation given there: 'A deputy $d$ in an organisation, having in mind to promote himself to top man by means of a coup, must first asses the power-structures as he sees them, or perhaps assess what subsets of the organisation must be deemed to be power-structures if a coup successful to him is to be found. There may be advantages, springing say from matters of secrecy or from national security, in finding the minimal such powerstructures allowing a successful coup in his favour. The quoted result shows that any minimal power-structure must give to at least one other deputy the status of a rival to $d$.' Any power-structure having $d$ as its only deputy has of course $d$ as its only contender, the only available successor to its current boss-presumably a desirable situation for $d$. There certainly exist power-structures with many deputies only one of whom is a contender; the theorem tells us that such power-structures are not minimal.

The proof uses the following result from [1] (Lemma 13, Theorem 14):

Lemma. Let $B, C \in \mathcal{P}$ and $\vee B=\vee C$. Then $B$ covers $C$ in $(\mathcal{P}, \subseteq)$ if and only if $B=C \cup\{z\}$ for some minimal member $z$ of $B$; and in that case

$$
t(B)=\text { either } t(C)+1 \text { or } t(C)
$$

according as there does or does not exist a topple set of $B$ containing $z$.

ProOf OF THE THEOREM: Let $M$ be a minimal power-structure in an organisation $X$, with $|M| \geqslant 2$. If $|M|=2$ then $M$ has the form $\{a, d\}$ with $a>d$, and $K=\{a\}$ is a power-structure having the same boss $a$ and stability 1 as $M$, contradicting the minimality of $M$. So in fact $|M| \geqslant 3$.

$M$ must have at least one contender; suppose it has exactly one, $c$ say.

Assume that $M$ has no deputy other than $c$. Then $M$ has the form

$$
M=\{a, c\} \cup E, \quad \text { where } a>c>e \text { for all } e \in E
$$

the only possible topple set is $\{a\}$, and $t(M)=1$. Since $E$ is not empty, $M$ has a minimal member other than $c$; let $y$ be one such, and write

$$
K=M \backslash\{y\}
$$

$K$ must be a power-structure with the same boss $a$ as $M$, since $y \neq a$ and $K$ is order-convex and an increasing subset of $M$. Since $K \subset M$ and $M$ covers $K$ in $\mathcal{P}$, the lemma gives $t(M)=t(K)$. This contradicts the minimality of $M$. Therefore $M$ 
has at least one deputy distinct from $c$. Let $z$ be a minimal member of $M$ which is $\leqslant c$ (there exists at least one). Suppose $z=c$. Then $M$ must be of the form

$$
M=\left\{a, c, d_{1}, d_{2}, \ldots, d_{n}\right\} \quad \text { for some } n \geqslant 1,
$$

where $a=\vee M$, and $c, d_{1}, d_{2}, \ldots, d_{n}$ are distinct deputies and therefore each minimal. For if some deputy, say $d_{1}$, were not minimal, then

$$
\left|M \backslash\left(\leqslant d_{1}\right)\right| \leqslant|M|-2<|M \backslash(\leqslant c)|,
$$

showing that the promotion of $c$ would involve removal of a set $M \backslash\{c\}$ of greater cardinality than would the promotion of $d_{1}$, contradicting the assumption that $c$ is a contender. But the form (2) inplies that all the deputies $d_{1}, d_{2}, \ldots, d_{n}$ are contenders, contradicting the assumption that $M$ has only the contender $c$.

Thus, $z<c$. Put

$$
J=M \backslash\{z\} .
$$

Let $d$ be a deputy of $M$ other than $c$. Since $d$ is not a contender,

$$
|M \backslash(\leqslant d)|>t(M)
$$

We cannot have $z=d$. If $z<d$ then $M \backslash(\leqslant d)=J \backslash(\leqslant d)$, so

$$
|J \backslash(\leqslant d)|>t(M)
$$

if $z \nless d$ then

$$
|J \backslash(\leqslant d)|=|M \backslash(\leqslant d)|-1 \geqslant t(M) ;
$$

so in either case,

$$
|J \backslash(\leqslant d)| \geqslant t(M)
$$

Now $t(J)=\min _{f}\{|J \backslash(\leqslant f)|: f$ is a deputy of $J\}$; the deputies of $J$ are precisely the deputies of $M$, including $c$, so

$$
t(J)=\min _{d}\{|J \backslash(\leqslant c)|, \quad|J \backslash(\leqslant d)|: d \text { is a deputy of } M, d \neq c\}
$$

and

$$
|J \backslash(\leqslant c)|=|M \backslash(\leqslant c)|=t(M)
$$

By (3) and (4),

$$
t(J)=t(M)
$$


But since $J \subset M$ and $J$ is a power-structure of $X$ and $\vee J=a=\vee M$, the minimality of $M$ gives $t(J)<t(M)$. This contradiction proves the theorem.

If a power-structure $B$ is not minimal, an induction argument shows that there must exist a power-structure $C$ covered by $B$ for which $\vee C=\vee B$ and $t(C)=t(B)$. By the lemma, $C^{\prime}=B \backslash\{z\}$ for some member $z$ which is minimal in $B$ and belongs to no topple set of $B$. The set of members which belong to no topple set is the set

$$
S=\{x \in B: x \leqslant c \text { for every contender } c \text { of } B\},
$$

called the safe core of $B$ since it is precisely these members who survive every possible coup in $B$. Thus any non-minimal power-structure has a nonempty safe core. The argument is reversible, so we have a characterisation of minimal power-structures:

TheOREM. A power-structure is minimal if and only if its safe core is empty.

Thus no one is safe in a minimal power-structure.

Corollary. Every power-structure which is a root system with two or more contenders is minimal. No chain is minimal.

If $B$ is a nonminimal power-structure with safe core $S$, then it is possible to obtain from it a minimal power-structure by deletion of part or all of $S$, that is, there exists a subset $S^{\prime}$ of $S$ such that $B^{\prime}:=B \backslash S^{\prime}$ is a minimal power-structure, with $t\left(B^{\prime}\right)=t(B)$.

\section{REFERENCES}

[1] J.B. Miller, 'Introduction to a theory of coups', Algebra Universalis $\theta(1979), 346-370$.

Department of Mathematics

Monash University

Clayton, Vic 3168

Australia 